K. Ichihara

Nagoya Math. J.

Vol. 87 (1982), 101-114

\title{
CURVATURE, GEODESICS AND THE BROWNIAN MOTION ON A RIEMANNIAN MANIFOLD I \\ RECURRENCE PROPERTIES
}

\author{
KANJI ICHIHARA
}

\section{§ 0. Introduction}

Let $M$ be an $n$-dimensional, complete, connected and locally compact Riemannian manifold and $g$ be its metric. Denote by $\Delta_{M}$ the Laplacian on $M$.

The Brownian motion on the Riemannian manifold $M$ is defined to be the unique minimal diffusion process $\left(X_{t}, \zeta, P_{x}, x \in M\right)$ associated to the Laplacian $\Delta_{M}$ where $\zeta$ is the explosion time i.e. if $\zeta(\omega)<+\infty, \lim _{t \rightarrow \zeta} X_{t}(\omega)$ $=\infty$. It should be remarked that $\zeta=+\infty$ a.s. if $M$ is compact. See Mckean [7], page 90.

The Brownian motion $X$ on $M$ is said to be recurrent if for every open subset $U$ of $M$

$$
P_{x}\left\{\omega \mid X_{t}(\omega) \in U \text { for some } t>0\right\}=1 \text { on } M ;
$$

otherwise it will be called transient.

It has been known that the Brownian motion on a compact Riemannian manifold is recurrent. See for example Mckean [7] page 99. In this paper we shall restrict our consideration to non compact case and clarify the relation between the recurrent and transient properties of the process $X$ and geodesics, curvature of $M$.

In the first section we shall summarize recurrence and transience of the Brownian motion on a rotationally symmetric Riemannian manifold (See Section 1 for the precise definition). Section 2 is devoted to the discussion for the general case. Some examples will be shown in the last section.

The author wishes to express his thanks to Professors H. Kunita and

Received July 23, 1980. 
H. Watanabe for their encouragement and interest in the preliminaries of this work.

\section{§1. Brownian motion on a model $M_{0}$}

Let us first give

Definition 1.1 (Greene and Wu [3]). A Riemannian manifold $R^{n}=$ $[0,+\infty) \times S^{n-1}$ given a metric $d r^{2}+g_{0}(r)^{2} d \theta^{2}$, is called a model $\left(M_{0}, g_{0}\right)$ where $g_{0}$ is a $C^{\infty}$ function on $[0,+\infty)$ which in addition satisfies: $g_{0}>0$ on $(0,+\infty), g_{0}(0)=0$ and $d g_{0} / d r(0)=1$ and $d \theta^{2}$ denotes the canonical metric on the unit sphere $S^{n-1}$.

Let $X_{t}^{0}=\left(r_{t}, \theta_{t}\right) \in[0,+\infty) \times S^{n-1}$ denote the Brownian motion on the model $\left(M_{0}, g_{0}\right)$. Then it is easy to see that recurrence and transience of the Brownian motion $X_{t}^{0}$ depend only on the behaviour of the radial process $r_{t}$ and that the radial part $r_{t}$ of $X_{t}^{0}$ is the unique one dimensional diffusion process on the interval $[0,+\infty)$ governed by the differential operator

$$
\frac{d^{2}}{d r^{2}}+\frac{(n-1)}{g_{0}(r)} \frac{d g_{0}(r)}{d r} \frac{d}{d r}
$$

Thus the next proposition easily follows from the criterion for the recurrence and transience of diffusions in one dimension.

Proposition 1.1. The following conditions are equivalent.

(i) The Brownian motion $X_{t}^{0}$ on a model $\left(M_{0}, g_{0}\right)$ is recurrent.

(ii) $\int^{+\infty} g_{0}(r)^{-n+1} d r=+\infty$.

It is to be noted that $g_{0}(r)^{n-1} d r d \theta$ is the volume element at a point $(r, \theta) \in M_{0}$.

In the remainder of this section we shall make some remarks on the model $\left(M_{0}, g_{0}\right)$.

Remark 1.1. Let $\left(M_{0}, g_{0}\right)$ be a model. Define

$$
K_{0}(r)=-\frac{1}{g_{0}(r)} \frac{d^{2} g_{0}(r)}{d r^{2}} .
$$

Let $\partial_{M_{0}}\left(x_{0}\right)$ denote the unit tangent vector at $x_{0}=(r, \theta)$ to the unique geodesic joining the point $x_{0}$ and the origin 0 . Then $K_{0}(r)$ corresponds with the sectional curvature of two dimensional planes at $x_{0}$ containing the vector $\partial_{M_{0}}\left(x_{0}\right)$. i.e. 


$$
K_{M_{0}}\left(\partial_{M_{0}}\left(x_{0}\right), X\right)=K_{0}(r) \quad \text { for every unit vector } X \in N\left(\partial_{M_{0}}\left(x_{0}\right)\right)
$$

where $N\left(\partial_{M_{0}}\left(x_{0}\right)\right)$ denotes the subspace of $T_{x_{0}}\left(M_{0}\right)$ orthogonal to the vector $\partial_{M_{0}}\left(x_{0}\right)$ and $K_{M_{0}}(X, Y), X, Y \in T_{x_{0}}\left(M_{0}\right)$ denotes the sectional curvature of $M_{0}$ for the two dimensional plane spanned by $X$ and $Y$. Hereafter $K_{0}(r)$ will be called the radial curvature of $M_{0}$. Furthermore it is also easy to see that the radial Ricci curvature $\operatorname{Ric}_{M_{0}}\left(\partial_{M_{0}}\left(x_{0}\right)\right)$ is equal to $(n-1) K_{0}(r)$. See Milnor [9] for the definitions of the sectional and Ricci curvatures.

Remark 1.2. Let $g_{0}^{i} i=1,2$ be two functions of the $C^{\infty}([0,+\infty))$ class such that for some $K_{0}^{i}(r) \in C^{\infty}([0,+\infty))$

$$
\frac{d^{2} g_{0}^{i}(r)}{d r^{2}}=-K_{0}^{i}(r) g_{0}^{i}(r) \quad \text { on }[0,+\infty)
$$

and

$$
g_{0}^{i}(0)=0, \quad \frac{d g_{0}^{i}}{d r}(0)=1
$$

The following comparison theorem is well known.

Sturm-Liouville Comparison Theorem. Let $g_{0}^{i}(r)$ be as above. Assume that for every $r \geqq 0$

$$
K_{0}^{1}(r) \leqq K_{0}^{2}(r)
$$

If $r_{0}$ is the first zero of $g_{0}^{2}(r)$, then $g_{0}^{1}(r) \geqq 0$ and $g_{0}^{1}(r) \geqq g_{0}^{2}(r)$ hold in the interval $r \in\left(0, r_{0}\right]$.

\section{§2. Brownian motion on a Riemannian manifold $M$}

In this section the Brownian motions on general Riemannian manifolds will be discussed. First of all let $K_{M}(\cdot, \cdot)$ and $\operatorname{Ric}_{M}(\cdot)$ denote the sectional and Ricci curvatures of $M$ respectively as in the first section.

A smooth curve on $M, m(r):[0, \ell(m)) \rightarrow M, \ell(m) \leqq+\infty$ is said to be a minimal geodesic if the following two conditions are satisfied.

(i) $m$ is a normal geodesic,

i.e.

$$
\nabla_{\dot{m}} \dot{m}=0 \text { and }\langle\dot{m}, \dot{m}\rangle=1 \text { on }[0, \ell(m)) .
$$

(ii) $d(m(0), m(r))=r \quad$ on $[0, \ell(m))$,

where $\nabla$ denotes the covariant differential, $\langle\cdot, \cdot\rangle$ is the inner product and $d(x, y), x, y \in M$ is the distance induced by the Riemannian metric $g$. See 
Milnor [9] for the details.

Under the above preparation, we can now state our main theorems.

THEOREM 2.1. If for some $p \in M$ there exists a model $\left(M_{0}, g_{0}\right)$ satisfying the following conditions (i) and (ii), then the Brownian motion $X$ on $M$ is recurrent.

(i) For every minimal geodesic $m(r):[0, \ell(m)) \rightarrow M, m(0)=p$,

$$
\operatorname{Ric}_{M}(\dot{m}(r)) \geqq(n-1) K_{0}(r) \quad \text { on }[0, \ell(m)) \text {. }
$$

(ii) $\int^{+\infty} g_{0}(r)^{-n+1} d r=+\infty$.

THEOREM 2.2. Let $M$ be simply connected. If for some $p \in M$ there exists a model $\left(M_{0}, g_{0}\right)$ satisfying the following two conditions (i) and (ii), then the Brownian motion $X$ on $M$ is transient.

(i) For every normal geodesic $m(r):[0,+\infty) \rightarrow M, m(0)=p$

$$
K_{M}(\dot{m}(r), X) \leqq K_{0}(r) \text { for every unit vector } X \in N(\dot{m}(r)) \text { on }[0,+\infty)
$$

(ii) $\int^{+\infty} g_{0}(r)^{-n+1} d r<+\infty$.

In order to prove the above theorems, we shall introduce some notations.

$$
\begin{aligned}
& \tau_{1}(\omega)=\inf \left\{t>0 \mid d\left(p, X_{t}(\omega)\right) \leqq 1\right\} \\
& \sigma_{\rho}(\omega)=\inf \left\{t>0 \mid d\left(p, X_{t}(\omega)\right) \geqq \rho\right\}, \quad \rho>1
\end{aligned}
$$

and

$$
\phi_{\rho}(x)=P_{x}\left[\tau_{1}<\sigma_{\rho}\right], \quad \phi_{\infty}(x)=P_{x}\left[\tau_{1}<\zeta\right] .
$$

Since $\lim \sigma_{\rho}(\omega)=\zeta(\omega), \lim \phi_{\rho}(x)=\phi_{\infty}(x)$ for each $x \in M$. Furthermore we set $\tilde{\phi}_{\rho}(\vec{x}) \stackrel{+\infty}{=} 1-E_{x}\left[e^{-\tau_{1} \wedge \wedge_{\rho}^{\rho+} \rho}\right]$

$$
\Gamma_{0, \rho}=\left\{x \in \partial\left(\Sigma_{\rho}-\bar{\Sigma}_{1}\right) \mid \varliminf_{\substack{y \in \sum_{\rho}-\bar{\Sigma}_{1} \\ y \rightarrow \bar{\phi}_{\rho}}} \tilde{\phi}_{\rho}(y)=0\right\}
$$

and

$$
\Gamma_{0, \infty}=\{x \in M \mid d(p, x)=1\} \cap \bigcup_{\rho>1} \Gamma_{0, \rho}
$$

where $\Sigma_{\rho}=\{x \in M \mid d(p, x)<\rho\}$.

The following proposition will be proved in the same way as in Ichihara [4], Chapter 2. 
Proposition 2.1. For each $\rho \in(1,+\infty]$

(i) $\phi_{\rho} \in C^{\infty}\left(\Sigma_{\rho}-\bar{\Sigma}_{1}\right)$ and $\Delta_{M} \phi_{\rho}=0$ in $\Sigma_{\rho}-\bar{\Sigma}_{1}$

(ii) $\phi_{\rho}(y) \stackrel{\tilde{\phi}_{\rho}}{\longrightarrow} g_{\rho}(x)=\left\{\begin{array}{l}1 \text { if } d(p, x)=1 \\ 0 \text { if } d(p, x)=\rho\end{array}\right.$ as $y \in \Sigma_{\rho}-\bar{\Sigma}_{1} \longrightarrow x \in \Gamma_{0, \rho}$

Here $\phi_{\rho}(y) \stackrel{\tilde{\phi}_{\rho}}{\rightarrow} g_{\rho}(x)$ means as follows. In case $\rho<+\infty, \phi_{\rho}\left(y_{n}\right) \rightarrow g_{\rho}(x)$ if $y_{n, n \geqq 1} \in \Sigma_{\rho}-\bar{\Sigma}_{1}$ tends to $x \in \Gamma_{0, \rho}$ and $\lim _{n \rightarrow \infty} \tilde{\phi}_{\rho}\left(y_{n}\right)=0$. In case $\rho=+\infty, \phi_{\infty}\left(y_{n}\right)$ $\rightarrow g_{\infty}(x)=1$ if $y_{n, n \geqq 1} \in\{x \in M: d(p, x)>1\}$ tends to $x \in \Gamma_{0, \infty}$ and $\lim _{n \rightarrow \infty} \tilde{\phi}_{\bar{\rho}}\left(y_{n}\right)$ $=0$ for every $\tilde{\rho} \in\left(1, \rho_{0}\right)$, with a positive constant $\rho_{0}=\rho_{0}(x)>1$.

We now introduce the Dirichlet integral on a bounded open subset $\Omega$ of $M$.

$$
D(\phi: \Omega)=\int_{\Omega}\langle\operatorname{grad} \phi, \operatorname{grad} \phi\rangle d V
$$

where $\phi \in C_{0}^{\infty}(\Omega)$, the set of infinitely differentiable, real valued functions with compact support in $\Omega$ and $d V$ is the Riemannian volume of $M$.

Define, for a fixed compact subset $K$ of $\Omega, H^{0,1}(\Omega, K)$ to be the closure of the set of functions $\phi \in C_{0}^{\infty}(\Omega)$ with $\phi \equiv 1$ on $K$.

The following lemma is a special case of the Dirichlet principle. See also Ichihara [4].

LEMma 2.1. For every $\rho \in(1,+\infty), \phi_{\rho} \in H^{0,1}\left(\Sigma_{\rho}, \bar{\Sigma}_{1}\right)$ and $\phi_{\rho}$ minimizes the Dirichlet integral

$$
D\left(\phi: \Sigma_{\rho}\right)=\int_{\Sigma_{\rho}}\langle\operatorname{grad} \phi, \operatorname{grad} \phi\rangle d V
$$

over $H^{0,1}\left(\Sigma_{\rho}, \bar{\Sigma}_{1}\right)$.

The following theorem is a fundamental criterion for recurrence and making use of Proposition 2.1 and Lemma 2.1, we can prove it by a similar argument to Ichihara [4].

THEOREM 2.3. The following two conditions are equivalent.

(i) The Brownian motion $X$ on $M$ is recurrent.

(ii) $\lim _{\rho \rightarrow+\infty} D\left(\phi_{\rho}: \Sigma_{\rho}\right)=0$.

Thus in order to prove Theorems 2.1 and 2.2 it suffices to verify the condition (ii) in Theorem 2.3. To do this we need some preparation from Riemannian geometry, which is summarized below.

Cut loci. (See Kobayashi and Nomizu [6] for the details) For $x \in M$, set 


$$
S_{x}=\left\{X \in T_{x}(M):\langle X, X\rangle=1\right\}
$$

where $T_{x}(M)$ is the tangent space at $x \in M$, and for $X \in S_{x}$.

$$
\begin{aligned}
& \mu_{x}(X)=\sup \left\{t>0: d\left(x, \exp _{x}(s X)\right)=s \text { for every } s \in(0, t)\right\} \\
& \tilde{C}(x)=\left\{\mu(X) X: X \in S_{x} \text { and } \mu(X)<+\infty\right\} \\
& C(x)=\exp _{x} \tilde{C}(x) .
\end{aligned}
$$

The sets $\tilde{C}(x)$ and $C(x)$ are called the cut loci for a point $x \in M$ in $T_{x}(M)$ and $M$ respectively.

The following properties will be used in the proof of our main theorems.

(A) The measure of $C(x)$ is zero.

(B) Set $E=\left\{t X: 0 \leqq t<\mu(x), X \in S_{x}\right\}$, then exp maps $E$ diffeomorphically onto an open subset $M \backslash C(x)$.

We are now in a position to prove Theorems 2.1 and 2.2.

Proof of Theorem 2.1. We first define the following functions

$$
\psi_{\rho}(r)=\left\{\begin{array}{cl}
\frac{\int_{r}^{\rho} g_{0}(s)^{-n+1} d s}{\int_{1}^{\rho} g_{0}(s)^{-n+1} d s} & \text { if } r \in(1, \rho) \\
1 & \text { if } 0 \leqq r \leqq 1 \\
0 & \text { otherwise }
\end{array}\right.
$$

and $\Psi_{\rho}(x)=\psi_{\rho}(d(p, x))$. Then it is obvious that

$$
\Psi_{\rho}(x)= \begin{cases}1 & \text { if } d(p, x) \leqq 1 \\ 0 & \text { if } d(p, x)=\rho\end{cases}
$$

and $\Psi_{\rho}(x)$ is Lipschiz continuous, and so $\Psi_{\rho} \in H^{0,1}\left(\Sigma_{\rho}, \bar{\Sigma}_{1}\right)$. Consequently applying Lemma 2.1, we obtain

(*) $\quad \int_{\Sigma_{\rho}}\left\langle\operatorname{grad} \phi_{\rho}, \operatorname{grad} \phi_{\rho}\right\rangle d V \leqq \int_{\Sigma_{\rho}}\left\langle\operatorname{grad} \Psi_{\rho}, \operatorname{grad} \Psi_{\rho}\right\rangle d V$.

Thus for the proof of Theorem 2.1, it is enough to show that the right hand side of $(*)$ tends to zero as $\rho \rightarrow+\infty$.

Let $C(p)$ be the cut locus of $p$. Note that we may assume $\mu(\theta) \geqq 1$ for every $\theta \in S^{n-1} \cong S_{p}$ without loss of generality. From the property (B) in "Cut loci", by the exponential map, we can pull back the Dirichlet integral $(*)$ to the tangent space $T_{p}(M)$ at $p$. 


$$
\int_{\Sigma_{\rho}}\left\langle\operatorname{grad} \Psi_{\rho}, \operatorname{grad} \Psi_{\rho}\right\rangle d V=\int_{S^{n-1}} d \theta \int_{1}^{\mu(\theta) \wedge \rho}\left(\frac{d \psi_{\rho}}{d r}\right)^{2} G(r, \theta) d r
$$

where $G(r, \theta)=\sqrt{\operatorname{det}\left(g_{i j}\right)(r, \theta)}, g=g_{i j} d x_{i} d x_{j}$ and $d \theta$ is the uniform measure on $S^{n-1}$.

On the other hand, from the assumption on curvature in Theorem 2.1, Laplacian comparison theorem, Greene and $\mathrm{Wu}$ [3] implies that for every $\theta \in S^{n-1}$ and $r \in(0, \mu(\theta))$

$$
\frac{1}{G(r, \theta)} \frac{\partial G(r, \theta)}{\partial r} \leqq \frac{(n--1)}{g_{0}(r)} \frac{d g_{0}(r)}{d r}
$$

From this, it is easy to show that with a positive constant $C$

$$
G(r, \theta) \leqq C \cdot g_{0}(r)^{n-1}
$$

for every $\theta \in S^{n-1}$ and $r \in[0, \mu(\theta))$. Combining all of this we have

$$
\begin{aligned}
\int_{\Sigma_{\rho}}\left\langle\operatorname{grad} \Psi_{\rho}, \operatorname{grad} \Psi_{\rho}\right\rangle d V & =\int_{S^{n-1}} d \theta \int_{1}^{\mu(\theta) \wedge \rho}\left(\frac{d \psi_{\rho}}{d r}\right)^{2} G(r, \theta) d r \\
& \leqq C \cdot \int_{S^{n-1}} d \theta \int_{1}^{\rho}\left(\frac{d \psi_{\rho}}{d r}\right)^{2} \cdot g_{0}(r)^{n-1} d r \\
& =\frac{C \cdot\left|S^{n-1}\right|}{\int_{1}^{\rho} g_{0}(r)^{-n+1} d r}
\end{aligned}
$$

which from the assumption (ii) converges to zero as $\rho \rightarrow+\infty$. Thus we have completed the proof of Theorem 2.1.

q.e.d.

Proof of Theorem 2.2. First note that under the assumption (i) in Theorem 2.2, Rauch comparison theorem gives the non existence of conjugate points to the point $p$. Therefore the exponential map at $p$ induces a diffeomorphism between $T_{p}(M)$ and $M$, because $M$ is simply connected. See Cheeger and Ebin [2], page 36, 37. Using this fact, we shall pull back the Dirichlet integral of $\phi_{\rho}$ to the tangent space $T_{p}(M)$.

i.e. $\quad \int_{\Sigma_{\rho}}\left\langle\operatorname{grad} \phi_{\rho}, \operatorname{grad} \phi_{\rho}\right\rangle d V=\int_{S^{n-1}} d \theta \int_{1}^{\rho}\left\langle\operatorname{grad} \phi_{\rho}, \operatorname{grad} \phi_{\rho}\right\rangle G(r, \theta) d r$ and it is easy to see that

$$
\geqq \int_{S^{n-1}} d \theta \int_{1}^{\rho}\left(\frac{\partial \phi_{\rho}}{\partial r}\right)^{2} G(r, \theta) d r .
$$

Now applying the Schwarz inequality, we obtain 


$$
\geqq \int_{S^{n-1}} d \theta \frac{1}{\int_{1}^{\rho} G^{-1}(r, \theta) d r} .
$$

On the other hand, under the assumption (i) of Theorem 2.2, Hessian comparison theorem, Greene and $\mathrm{Wu}$ [3] gives that for every $(r, \theta) \in(0,+\infty)$ $\times S^{n-1}$

$$
\frac{1}{G(r, \theta)} \frac{\partial G(r, \theta)}{\partial r} \geqq \frac{(n-1)}{g_{0}(r)} \frac{d g_{0}(r)}{d r}
$$

Consequently we have, with a positive constant $C$

$$
G(r, \theta) \geqq C \cdot g_{0}(r)^{n-1}
$$

for every $(r, \theta) \in[0,+\infty) \times S^{n-1}$. Thus we get

$$
\begin{aligned}
\varliminf_{\rho \rightarrow+\infty} \int_{\Sigma_{\rho}}\left\langle\operatorname{grad} \phi_{\rho}, \operatorname{grad} \phi_{\rho}\right\rangle d V & \geqq \varliminf_{\rho \rightarrow+\infty} \int_{S^{n-1}} d \theta \frac{1}{\int_{1}^{\rho} G^{-1}(r, \theta) d r} \\
& \geqq \frac{C \cdot\left|S^{n-1}\right|}{\int_{1}^{\infty} g_{0}(r)^{-n+1} d r}>0
\end{aligned}
$$

from the assumption (ii). This together with Theorem 2.3 completes the proof.

q.e.d.

\section{§3. Examples}

In this section we shall show several examples to illustrate our theorems.

1. (Two dimensional case) Before showing our examples, we shall make a remark. Let $M$ be a two dimensional, $C^{\infty}$, simply connected, non compact Riemannian manifold. It follows from complex function theory that $M$ is conformally diffeomorphic either to the complex plane or to the unit disk in the complex plane. (See for example Springer [10].) In the first case one says that $M$ is parabolic, in the second case hyperbolic.

In connection with probability theory, Kakutani [5] has proven that the Brownian motion on $M$ is recurrent if and only if the manifold $M$ is parabolic.

1.1. Let $M$ be a two dimensional (not necessarily simply connected) Riemannian manifold and $K(x), x \in M$ be the Gaussian curvature of the 
Riemannian manifold. The following proposition which is essentially due to Blanc and Fiala [1] is obtained as a corollary to Theorem 2.3.

Proposition 3.1. If the total absolute curvature is finite

i.e.

$$
\int_{M}|K(x)| d V(x)<+\infty
$$

then the Brownian motion $X$ on $M$ is recurrent.

Proof. From the property (B) of the cut locus, we have that relative to geodesic polar coordinates $(r, \theta)$ centered at a fixed point $p \in M$, the Riemannian metric is of the form $d r^{2}+g(r, \theta)^{2} d \theta^{2}$ in $\left\{(r, \theta) \in(0,+\infty) \times S^{1}\right.$ : $r \in(0, \mu(\theta))\}$ and so

$$
\int_{M}|K(x)| d V(x)=\int_{M \backslash C(p)}|K(x)| d V(x)=\int_{S^{1}} d \theta \int_{0}^{\mu(\theta)}|K(r, \theta)| g(r, \theta) d r
$$

by the property (A). On the other hand, the Gaussian curvature $K(r, \theta)$ at $(r, \theta), \theta \in S^{1}, r \in[0, \mu(\theta))$ is represented in the following way. See Struik [11].

$$
K(r, \theta)=-\frac{1}{g(r, \theta)} \frac{\partial^{2} g(r, \theta)}{\partial r^{2}}
$$

Therefore we obtain

$$
\int_{M}|K(x)| d V(x)=\int_{S} d \theta \int_{0}^{\mu(\theta)}\left|\frac{\partial^{2} g(r, \theta)}{\partial r^{2}}\right| d r<+\infty
$$

Set

$$
C(\theta)=\int_{0}^{\mu(\theta)}\left|\frac{\partial^{2} g(r, \theta)}{\partial r^{2}}\right| d r<+\infty \quad \text { a. } a . \theta \in S^{1}
$$

Then for $r<\mu(\theta)$,

$$
\left|\frac{\partial g(r, \theta)}{\partial r}\right| \leqq\left|\int_{0}^{r} \frac{\partial^{2} g(s, \theta)}{\partial s^{2}} d s\right|+\left|\frac{\partial g(0, \theta)}{\partial r}\right| .
$$

Since $\partial g(0, \theta) / \partial r \equiv 1$, Struik [11], it follows

$$
\leqq \int_{0}^{r}\left|\frac{\partial^{2} g(s, \theta)}{\partial s^{2}}\right| d s+1 \leqq C(\theta)+1
$$

Consequently from the fact $g(0, \theta)=0$, we get 


$$
g(r, \theta) \leqq \int_{0}^{r}\left|\frac{\partial g(s, \theta)}{\partial s}\right| d s \leqq(C(\theta)+1) r
$$

We now define

$$
\begin{gathered}
\psi_{\rho}(r)=\left\{\begin{array}{cc}
\frac{\log (\rho / r)}{\log \rho}, & r \in(1, \rho) \\
1, & r \leqq 1
\end{array}\right. \\
\Psi_{\rho}(x)=\psi_{\rho}(d(p, x)) .
\end{gathered}
$$

Then by the same reasoning as in the proof of Theorem 2.1, it follows that

$$
\begin{aligned}
\int_{\Sigma_{o}}\left\langle\operatorname{grad} \phi_{\rho}, \operatorname{grad} \phi_{\rho}\right\rangle d V & \leqq \int_{\Sigma_{\rho}}\left\langle\operatorname{grad} \Psi_{\rho}, \operatorname{grad} \Psi_{\rho}\right\rangle d V \\
& =\int_{S^{1}} d \theta \int_{1}^{\mu(\theta) \wedge \rho}\left(\frac{d \psi_{\rho}(r)}{d r}\right)^{2} g(r, \theta) d r \\
& =\int_{S^{1}} d \theta \int_{1}^{\mu(\theta) \wedge \rho} \frac{1}{r^{2}} g(r, \theta) d r /(\log \rho)^{2} \\
& \leqq \int_{S^{1}} d \theta \int_{1}^{\rho}(1+C(\theta)) \frac{1}{r} d r /(\log \rho)^{2} \\
& =\left\{2 \pi+\int_{M}|K(x)| d V(x)\right\} \cdot \frac{1}{\log \rho} .
\end{aligned}
$$

which goes to zero as $\rho \rightarrow+\infty$. This implies recurrence of the process $X$.

1.2. Let $M$ be a two dimensional Riemannian manifold and $K(x)$ denote the Gaussian curvature of $M$ as in Example 1.

(i) If for some $p \in M$

$$
K(x) \geqq-\frac{1}{d(p, x)^{2} \cdot \log d(p, x)}
$$

holds outside of a compact subset of $M$, then the Brownian motion $X$ on $M$ is recurrent.

(ii) Assume that $M$ is simply connected and that its Gaussian curvature $K(x)$ is nonpositive everywhere. If for some $p \in M$ and a positive constant $\varepsilon$

$$
K(x) \leqq-\frac{1+\varepsilon}{d(p, x)^{2} \log d(p, x)}
$$

holds outside of a compact subset of $M$, then the Brownian motion $X$ on $M$ is transient. 
It should be remarked that the result (i) is a generalization of Milnor [8] and Greene and $\mathrm{Wu}$ [3] and (ii) is due to them.

Proof of (i). In order to prove (i), it suffices to construct a model $\left(M_{0}, g_{0}\right)$ which satisfies the conditions (i) and (ii) of Theorem 2.1. To do this, we first define a function $K_{0}(r) \in C^{\infty}([0,+\infty))$ nonpositive as follows.

(i) With a positive constant $C$

$$
\begin{gathered}
K_{0}(r)=-\frac{1}{r^{2} \log r} \quad \text { on }[C,+\infty) . \\
K_{0}(d(p, x)) \leqq K(x) \quad \text { on } M .
\end{gathered}
$$

Let $g_{0}(r) \in C^{\infty}([0,+\infty))$ be the unique solution of the following Jacobi equation.

$$
\left\{\begin{array}{l}
\frac{d^{2} g_{0}(r)}{d r^{2}}=-K_{0}(r) g_{0}(r) \\
g_{0}(0)=0, \frac{d g_{0}}{d r}(0)=1
\end{array}\right.
$$

Then from the assumptions on $K(r)$ and the Strum Comparison Theorem, it follows that $g_{0}(r)>0$ for $r>0$. Thus we have obtained a model $\left(M_{0}, g_{0}\right)$ which obviously satisfies the condition (i) in Theorem 2.1. The integral condition (ii) of Theorem 2.1 will be verified following the argument of Milnor [8].

Let

$$
\begin{aligned}
& g_{1}(r)=r \log r, \quad r \geqq C \text { and } \\
& K_{1}(r)=-\frac{1}{g_{1}(r)} \frac{d^{2} g_{1}(r)}{d r^{2}}=-\frac{1}{r^{2} \log r} .
\end{aligned}
$$

Multiplying a small positive constant if necessary, we may assume

$$
\begin{aligned}
& g_{1}(C)>g_{0}(C) \\
& \frac{d g_{1}}{d r}(C)>\frac{d g_{0}}{d r}(C) .
\end{aligned}
$$

Suppose that there exists a number $C_{1} \in(C,+\infty)$ such that

$$
g_{1}(r)>g_{0}(r), \quad r \in\left[C, C_{1}\right)
$$

and

$$
g_{1}\left(C_{1}\right)=g_{0}\left(C_{1}\right)
$$


Then for $r \in\left[C, C_{1}\right]$

$$
\frac{d^{2} g_{0}(r)}{d r^{2}}=-K_{0}(r) g_{0}(r)=-K_{1}(r) g_{0}(r) \leqq-K_{1}(r) g_{1}(r)=\frac{d^{2} g_{1}(r)}{d r^{2}}
$$

Integrating both sides, we have for $r \in\left[C, C_{1}\right]$

$$
\frac{d g_{0}(r)}{d r}-\frac{d g_{0}(C)}{d r} \leqq \frac{d g_{1}(r)}{d r}-\frac{d g_{1}(C)}{d r}
$$

and so from the above assumption $(* *)$

$$
\frac{d g_{0}(r)}{d r}<\frac{d g_{1}(r)}{d r} \quad \text { for } r \in\left[C, C_{1}\right]
$$

Integrating this inequality, we get for $r \in\left[C, C_{1}\right]$.

$$
g_{0}(r)-g_{0}(C)<g_{1}(r)-g_{1}(C) .
$$

Consequently it follows from the assumption $(*)$ that

$$
g_{0}(r)<g_{1}(r)
$$

for any $r \in\left[C, C_{1}\right]$, which is a contradiction. Thus we have shown that

$$
g_{0}(r)<g_{1}(r)=r \log r \quad \text { for every } r>C .
$$

Since $\int^{+\infty} \frac{d r}{r \log r}=+\infty$, it also follows that

$$
\int^{+\infty} \frac{d r}{g_{0}(r)}=+\infty
$$

q.e.d.

The proof of (ii) will be done in a similar way to that of (i), using the test function $g_{1}(r)=r \cdot(\log r)^{1+\varepsilon / 2}$ instead.

3. Let $M$ be an $n(\geqq 3)$ dimensional, complete, connected Riemannian manifold. Then we have

(i) If for some $p \in M$ and a positive constant $\varepsilon$,

$$
\text { "the Ricci curvature at } x " \geqq \frac{(n-1)+\varepsilon}{4} \cdot \frac{1}{d(p, x)^{2}}
$$

holds outside of a compact subset of $M$, then the Brownian motion $X$ on $M$ is recurrent.

(ii) Suppose that $M$ is simply connected and that the sectional 
curvature of $M$ is nonpositive. Then the Brownian motion $X$ on $M$ is transient.

The proofs of the above results are carried out by the same comparison methods as in Example 2. We only note that the test functions $g_{1}(r)=$ $(\log r)^{k}$ with a sufficiently large $k$ and $g_{1}(r)=r$ will be used instead respectively.

4. We consider an embedded $n$-dimensional hypersurface $S_{n}$ in $R^{n+1}$ defined by

$$
S_{n}: x_{n+1}=f\left(x_{1}, \cdots, x_{n}\right) .
$$

For the surface we have

(i) $n=2$.

(A) For $f\left(x_{1}, x_{2}\right)=x_{1}^{2}-x_{2}^{2}$, the Brownian motion $X$ on $S_{2}$ is recurrent.

(B) If $f$ is a radial function, then the Brownian motion on $S_{2}$ is recurrent.

(ii) $n \geqq 3$. Suppose $f$ is a radial function.

(A) If for all sufficiently large $x \in R^{n}$,

$$
\left|\frac{d f}{d|x|}\right| \geqq C \cdot\left(\frac{|x|^{n-2}}{\log |x|}\right)
$$

with a positive constant $C$, then the Brownian motion $X$ on $S_{n}$ is recurrent.

(B) If for a positive constant $\varepsilon$

$$
\left|\frac{d f}{d|x|}\right| \leqq 0\left(\frac{|x|^{n-2}}{(\log |x|)^{1+\varepsilon}}\right)
$$

then the Brownian motion $X$ on $S_{n}$ is transient.

In order to prove the case (i), (A), it suffices making use of the Gaussian curvature

$$
K\left(x_{1}, x_{2}\right)=\frac{f_{x_{1} x_{2}} f_{x_{2} x_{2}}-f_{x_{1} x_{2}}^{2}}{\left(f_{x_{1}}^{2}+f_{x_{2}}^{2}+1\right)^{2}}
$$

to verify that the total absolute curvature is finite.

The proof of the cases (i), (B) and (ii) proceeds as follows. Since $f$ is a radial function, with polar coordinates $(r, \theta)$ of $R^{n}$, we have

$$
d x_{1}^{2}+\cdots+d x_{n}^{2}+d x_{n+1}^{2}=d r^{2}+r^{2} d \theta^{2}+f_{r}^{2} d r^{2} .
$$

Set 


$$
p(r)=\int_{0}^{r} \sqrt{1+f_{u}^{2}} d u \text { and } s=p(r) .
$$

Then $S_{n}$ is a model with geodesic polar coordinates $(s, \theta)$ and its Riemannian metric has the form

$$
d s^{2}+\left(p^{-1}(s)\right)^{2} d \theta^{2}
$$

where $p^{-1}$ is the inverse function of $p$. On the other hand, since

$$
\int^{+\infty} \frac{1}{\left(p^{-1}(s)\right)^{n-1}} d s=\int^{+\infty} \frac{\sqrt{1+f_{r}^{2}}}{r^{n-1}} d r
$$

the conclusions follow from Proposition 1.

\section{REFERENCES}

[1] C. Blanc and F. Fiala, Le type d'une surface et sa courbure totale, Comment. Math. Helv., 14 (1941-42), 230-233.

[2] J. Cheeger and D. G. Ebin, Comparison theorems in Riemannian geometry, NorthHolland Pub. Co., 1975.

[ 3 ] R. E. Greene and H. Wu, Function theory on manifolds which posses a pole, Lecture notes, Springer, No. 699.

[4] K. Ichihara, Some global properties of symmetric diffusion processes, Publ. R.I.M.S., Kyoto Univ., 14, No. 2, (1978), 441-486.

[5] S. Kakutani, Random walk and the type problem of Riemann surfaces, Princeton Univ. Press 1961, 95-101.

[6] S. Kobayashi and K. Nomizu, Foundations of differential geometry II, Interscience, 1969.

[ 7 ] H. P. Mckean, Stochastic integrals, Academic press, 1969.

[8] J. Milnor, On deciding whether a surface is parabolic or hyperbolic, Amer. Math. Monthly, 84 (1977), 43-46.

[9] - Morse theory, Princeton Univ. Press, 1963.

[10] G. Springer, Introduction to Riemann surfaces, Addison-Wesley, Reading, Mass., 1950.

[11] D. Struik, Lectures on classical differential geometry, Addison-Wesley, Reading, Mass., 1950.

Department of Applied Science

Faculty of Engineering

Kyushu University

Fukuoka, Japan

Current address:

Department of Mathematics

Faculty of General Education

Nagoya University

Nagoya, Japan 